\title{
Die gesellschaftliche Verantwortung von Pharmaunternehmen für die Produktion, Preisgestaltung und Allokation von Covid-19- Impfstoffen
}

\section{Einleitung}

Im vorliegenden Beitrag diskutiere ich die besondere Verantwortung der pharmazeutischen Industrie - bzw. die Verantwortung der in dieser Industrie agierenden Unternehmen - im Kontext der Covid-19-Pandemie ${ }^{1}$, insbesondere in Hinblick auf die Produktion, Preisgestaltung und Allokation von entsprechenden Impfstoffen. ${ }^{2}$ Pharmaunternehmen tragen in der vom sogenannten Corona-Virus ausgelösten Gesundheits-, Wirtschafts- und Gesellschaftskrise eine besonders große Verantwortung. Sie allein verfügen über die Kombination von wissenschaftlichem und technologischem Know-how sowie über die notwendigen Produktions- und Logistikkapazitäten, um in absehbarer Zeit Impfstoffe gegen das Virus zu entwickeln, diese in großer Zahl zu produzieren und anschließend $\mathrm{zu}$ distribuieren. Abgesehen von Fragen bezüglich der Entwicklung dieser Impfstoffe (vgl. Scholz/ Smith 2020) ergeben sich vor dem Hintergrund der zu erwartenden Knappheit dieser Impfstoffe unternehmensethisch relevante Fragen, insbesondere zur anschließenden Preisgestaltung und Allokation. Von besonderer Relevanz sind hier die folgenden zwei Fragen: (1) Wie erfolgt die Preisgestaltung dieser Impfstoffe? (2) Welche Personen oder Personengruppen sollen diese Impfstoffe vorrangig erhalten? Keine dieser konkreten Fragen kann ich im vorliegenden Artikel beantworten. Stattdessen skizziere und kritisiere ich hier allgemeiner das mögliche Szenario einer Preisgestaltung und Allokation der >Corona-Impfstoffe nach ausschließlich markwirtschaftlichen Kriterien.

Im Folgenden werde ich argumentieren, dass die Bekämpfung der Covid-19Krise mithilfe von Impfstoffen bzw. die konkrete Preisgestaltung und Allokation dieser Impfstoffe neben den gesundheitlichen Implikationen auch erheblichen Einfluss auf die Verfestigung der globalen Ungleichheit nehmen kann. Vor dem Hintergrund eines sich zunehmend ausbreitenden Nationalismus, dem partiellen

1 Der in diesem Artikel dargestellte Wissensstand entspricht dem vom Frühsommer 2020. Weitere Entwicklungen, bspw. Reaktionen seitens der pharmazeutischen Industrie auf diesen oder frühere Artikel des Autors zum gleichen Thema (bspw. Scholz/Smith 2020) sowie neuere politische Entwicklungen etc., können hier entsprechend keine Beachtung finden.

2 Ich gehe in diesem Artikel davon aus, dass Unternehmen moralische Subjekte sind. Vgl. diesbezüglich `Unternehmen als moralische Akteure< (Neuhäuser 2011) und zu einer entsprechenden Kontroverse die Beiträge in >The Moral Responsibility of Firms` (Orts/ Smith 2017). 
Scheitern globaler Institutionen und der sich daraus ergebenden sogenannten 'Governance-Lücker auf globaler Ebene tragen Pharmaunternehmen eine gesellschaftliche und quasipolitische Mitverantwortung für eine gerechte Preisgestaltung und Allokation der dringend benötigten Impfstoffe. Welche Personen oder Personengruppen wann und zu welchem Preis Zugang zu Impfstoffen erhalten, darf nicht ausschließlich mittels preisinduzierter Marktmechanismen noch allein von Nationalstaaten oder individuell von Unternehmen entschieden werden. Preis und Vergabe sollten stattdessen und trotz der diesbezüglichen Herausforderungen (beispielsweise Uneinigkeit der Staatengemeinschaft, Geschwindigkeit des Prozesses) global durch supranationale Institutionen mithilfe von Multi-StakeholderDialogen koordiniert werden. Die Unternehmen der Pharmaindustrie sollten sich an diesem Dialog beteiligen und etwaige Institutionen, die diesen beherbergen können, aktiv unterstützen - dies liegt nicht nur in ihrer gesellschaftlichen Verantwortung als globale Corporate Citizen, sondern, wie ich im Folgenden argumentieren werde, gleichermaßen in ihrem Eigeninteresse.

\section{Covid-19-Pandemie: Globale Gesundheits-, Wirtschafts- und Gesellschaftskrise}

Die Covid-19-Pandemie löste zunächst die größte globale Gesundheitskrise seit Beendigung des Zweiten Weltkrieges mit weltweit Hunderttausenden von Toten und einem teil- und zeitweisen Zusammenbruch nationaler Gesundheitssysteme aus. Mit dieser Krise geht eine ebenfalls historische globale Wirtschaftskrise einher. Ausgelöst durch die aus medizinischer und epidemiologischer Perspektive notwendigen Einschränkungen kam es zu einer globalen Rezession, einem immensen Anstieg der globalen Arbeitslosenzahlen sowie hohen Unsicherheiten und Volatilitäten auf den Finanzmärkten. Das Ausmaß dieser ökonomischen Krise kann bisher nur durch gewaltige Investitionen seitens der Nationalstaaten und supranationalen Institutionen (beispielsweise der Europäischen Union, der Europäischen Zentralbank oder der Weltbank) einigermaßen unter Kontrolle gehalten werden. Während die medizinischen und ökonomischen Auswirkungen der Pandemie bzw. adäquate Reaktionen darauf fast umgehend Eingang in die öffentliche Debatte fanden, entwickelte sich erst zeitversetzt ein Bewusstsein und eine öffentliche Diskussion hinsichtlich ihrer gesellschaftlichen Folgen (vgl. Loerzer 2020; Wimalasena 2020; Klingst 2020; Fisher/Bubola 2020). Die von Regierungen zur Eindämmung der Pandemie erlassenen (und m. E. notwendigen Maßnahmen) bewirken eine nicht unerhebliche Einschränkung von Grundrechten, beispielsweise der Bewegungs- und Versammlungsfreiheit. Gleichzeitig wurde der direkte Zugang zu Kultur- und Bildungseinrichtungen de facto unmöglich gemacht und wird auch auf unabsehbare Zeit erheblich erschwert bleiben. In der Summe dieser Einschränkungen erkenne ich die Anzeichen einer Gesellschaftskrise. 


\section{Rückkehr zur bisherigen Normalität nur durch Impfungen möglich}

Eine Rückkehr zu einer Normalität, wie wir sie vor der Krise kannten, ist erst nach Erreichung einer sogenannten `Herdenimmunität` möglich, die aller Voraussicht nach nur mithilfe einer Immunisierung durch Impfstoffe erreichbar ist. Weltweit forschen zahlreiche Unternehmen in derzeit mehr als hundert Projekten an der Entwicklung dieser Impfstoffe, wobei nur ca. 20 bis 30 Projekten eine höhere Erfolgswahrscheinlichkeit zugestanden wird (vgl. WHO 2020a). Zu dem Zeitpunkt, an dem einem Unternehmen die Entwicklung eines Impfstoffes gelingt, entsteht ein unmittelbarer milliardenfacher globaler Bedarf (vgl. Mullard 2020; Usher 2020; Khamsi 2020). Dieser Bedarf wird auf ein relativ kleines Angebot treffen: Selbst einer der global größten Impfstoffhersteller, das französische Unternehmen Sanofi, könnte über alle - also auch über Covid-unabhängige - Impfstoffgruppen hinweg lediglich eine Milliarde Einheiten pro Jahr produzieren - vorausgesetzt, dieses Unternehmen würde jegliche bisherigen Impfstoffproduktionen, beispielsweise für Diphtherie, Hepatitis, Keuchhusten usw., einstellen (vgl. Le Ker/ Müller 2020). Bei einer ungefähren Weltbevölkerung von ca. acht Milliarden Menschen und einer angenommenen Einmalimpfung dauerte es demnach ungefähr acht Jahre, bis weltweit jeder Mensch eine Impfstoffdosis erhalten könnte. ${ }^{3}$

\section{Verfestigung der globalen und sozialen Ungleichheit mittels der Vergabe von Impfstoffen durch Marktmechanismen}

Aus dieser absehbaren Knappheit der Impfstoffe resultiert prinzipiell die Möglichkeit exorbitanter Preisforderungen des produzierenden Unternehmens. Es ist nicht ausgeschlossen, dass sich ein grundsätzlich aus Angebot und Nachfrage bestimmter Preis letztendlich aus einem Bestbieterverfahren ergibt ${ }^{4}$ : Nationalstaaten würden Gebote für kleinere oder größere Chargen der Impfstoffe abgeben. Komplettiert werden könnte dieses Verfahren durch Gebote großer Stiftungen, Konzerne und durch einige sogenannte Superreiche. In diesem Szenario würden zunächst die reichen Industrieländer, anschließend Schwellenländer und zuletzt die sogenannten Entwicklungsländer Zugang zu den Impfstoffen erhalten. Sollten in allen oder

3 Bei dieser Kalkulation (ca. 8 Milliarden Weltbevölkerung / 1 Milliarde produzierte Impfstoffdosen p. a.) handelt es sich lediglich um ein Beispiel. Die tatsächlich benötigten Impfdosen könnten geringer sein, da etwa Kinder aufgrund der fast symptomlosen Krankheitsverläufe möglicherweise nicht geimpft werden müssten und da gleichzeitig weitere Therapieformen entwickelt werden. Andererseits könnte die benötigte Anzahl der Impfdosen auch höher sein, wenn beispielsweise Mehrfachimpfungen notwendig sind. Die Geschwindigkeit der Produktion hängt außerdem maßgeblich von der Form des Impfstoffes ab (vgl. Khamsi 2020; Le Ker/Müller 2020; Mullard 2020). Vgl. hierzu auch die Forderungen von >The people's vacciner (UNAIDS 2020).

4 Vgl. hierzu auch die Debatte um das anscheinend gegen Covid-19 wirksame Medikament Remdesivir (vgl./ Garde/Silveman 2020). 
auch nur in einigen dieser Länder ebenfalls marktwirtschaftliche Kriterien dominieren, ein Verkaufspreis der Impfstoffe also über Angebot und Nachfrage bestimmt werden, erhielten wiederum zunächst Reiche und dann abstufend Ärmere und Arme Zugang zu den Impfstoffen. ${ }^{5}$

Eine solche Preis- und Vergabepolitik erhöht - neben den gesundheitlichen Implikationen - auch die Gefahr einer sich weiter verfestigenden und u. U. wachsenden globalen und sozialen Ungleichheit. Durch den kaufkraftbedingt bevorzugten Zugang zu Impfstoffen könnten reiche Nationalstaaten ihre Ökonomien schneller wieder aktivieren als ärmere Staaten und erhielten dadurch erhebliche globale Wettbewerbsvorteile. Die BürgerInnen dieser reichen Staaten kämen entsprechend schneller wieder in den Genuss von Freiheitsrechten (zum Beispiel Bewegungs- und Versammlungsfreiheit) als die BürgerInnen ärmerer Staaten, könnten schneller wieder vollumfänglich einer Erwerbsarbeit nachgehen, stärker konsumieren, hätten schneller wieder direkten Zugang zu Kultur- und vor allem zu Bildungs- und Ausbildungsinstitutionen. Diese zwischen Nationalstaaten bestehenden Tendenzen zur Verstetigung und Verschärfung der globalen Ungleichheit würden sich bei einer preisgesteuerten Vergabe von Impfstoffen auch innerhalb der Staaten fortsetzen und dort ähnliche Auswirkungen auf die soziale Ungleichheit haben. Sollte innerhalb eines Staates der Zugang zu Impfstoffen Preisbarrieren unterliegen, erhielten zunächst reiche BürgerInnen Impfstoffe. Diese kämen entsprechend schneller wieder in den Genuss ihrer Grundrechte und könnten umfänglich ihrer Erwerbsarbeit mit all den damit verbundenen Vorteilen nachgehen. Es wären die Kinder dieser wohlhabenden Eltern, die umfänglicheren und direkteren Zugang zu Kultur-, Ausbildungs- und Bildungsinstitutionen hätten. Gleichzeitig könnten beide Elternteile - auch die immer noch geringer bezahlten und häufig mit der Kinderbetreuung beschäftigten Frauen - ihren jeweiligen Karrieren (mit allen positiven ökonomischen Konsequenzen) nachgehen.

\section{Individuelle Unternehmensverantwortung und globales Governance Gap}

Die dargelegten Beispiele illustrieren einige Konsequenzen der ausschließlich an marktwirtschaftlich, also letztlich durch Preise, koordinierten Vergabe von Impfstoffen: Der ressourcenabhängige Zugang zu Impfstoffen verfestigt und verschärft unter Umständen die bestehende globale und soziale Ungleichheit. Ein Unternehmen, welches vor dem Hintergrund des oben angeführten globalen Governance Gaps zulässt, dass die Preisbildung und Allokation eines Sars-Cov-2-Impfstoffes ausschließlich über Marktmechanismen (Angebot und Nachfrage; Preise) koordiniert wird, trägt entsprechend eine Mitverantwortung an dieser sich verstetigenden Ungleichheit.

5 Das letzte Szenario wird weniger in den reichen und durch Krankenkassen versorgten Industrieländern und häufiger in ärmeren Schwellenländern zu erwarten sein. 
Es kann hinterfragt werden, ob Unternehmen eine Verantwortung zur Beseitigung von gesellschaftlichen Problemen, hier also von Ungleichheit, tragen. Skeptiker dieser Position werden auf das von Milton Friedman (1962; 1970) popularisierte und in verschiedenen Versionen weitergedachte Argument einer Arbeitsteilung zwischen Staaten und Unternehmen hinweisen. Demzufolge wäre es Aufgabe der individuellen Nationalstaaten und eventuell supranationaler Institutionen, gesellschaftliche Phänomene wie soziale und globale Ungleichheit zu adressieren und diese mithilfe von Regularien, Anreizen und Sanktionen abzubauen. Ein Pharmaunternehmen, das über einen >Corona-Impfstoff ‘ verfügt, wäre in diesem Szenario lediglich Regelbefolger, hätte aber keine weitere (politische) Verantwortung für die Konsequenzen der angewandten Preis- und Vergabepolitik, solange es sich an die bestehenden Gesetze hält (vgl. Friedman 1962; 1970).

Dieses Argument erscheint mir in der gegenwärtigen Situation aus zwei Gründen nicht haltbar zu sein: Zunächst handelt es sich bei der Covid-19-Pandemie um ein globales Phänomen. Das Virus befällt - zumindest nach aktuellem Wissensstand - die Menschen aller fünf Kontinente gleichermaßen. Die durch eine preisgesteuerte Vergabe sich verfestigende oder sogar verstärkte Ungleichheit ist ebenfalls global. Demgegenüber steht ein globales Governance Gap: Es fehlt de facto an einer Institution, die den Einkauf und die Vergabe der Impfstoffe koordinieren kann. Bestehende Institutionen, die diese Rolle übernehmen könnten, beispielsweise die Weltgesundheitsorganisation (WHO), werden derzeit systematisch von einigen Staaten unterminiert. Weiterhin lassen sich im Kontext der Pandemie zahlreiche nationale Alleingänge beobachten. Diese reichen von der kläglich unzureichenden Solidarität und Abstimmung der europäischen Länder zur Hochzeit der Krise - Grenzen wurden unkoordiniert geschlossen, Hilfsanfragen verweigert und Lieferungen mit Schutzausrüstungen kompetitiv erworben oder sogar illegal einbehalten (vgl. Becker/Müller 2020; Landfried 2020) - über die Versuche der USA, Biotechnologieunternehmen $\mathrm{zu}$ erwerben, welche erfolgversprechende Resultate zu Impfstoffentwicklungen vorweisen können (vgl. Dostert 2020), bis hin zum starken Lobbying einiger Länder, große Chargen möglicher Impfstoffe und Medikamente gegebenenfalls früher zu erhalten als andere (vgl. Mintzes/ Hoen 2020; Bubrowski 2020).

Unter diesen von mangelnder Solidarität auf nationalstaatlicher Ebene und dem Fehlen einer globalen Regulierungsinstanz geprägten Bedingungen können sich Unternehmen nicht auf eine Position der Regelbefolgung zurückziehen. Konkret bedeutet dies Folgendes: Wenn ein Unternehmen einen Impfstoff entwickelt hat, wird die Vergabe dieses Impfstoffes nicht nur Einfluss auf den Gesundheitszustand der EmpfängerInnen haben, sondern ebenso gesellschaftliche Auswirkungen, insbesondere in Hinblick auf Ungleichheitsthematiken, hervorrufen. Vor dem Hintergrund eines globalen Governance Gaps trägt dieses Unternehmen eine erhebliche und nur schwer zu delegierende politische Verantwortung. Gleichzeitig dürfen Entscheidungen hinsichtlich der Vergabe des Impfstoffes, wenn sie nicht über den Preismechanismus geregelt werden, nicht von diesem Unternehmen 
allein getroffen werden. Für die weitreichende Entscheidung, welche Personen oder Personengruppen zu welchem Zeitpunkt welche Volumina erhalten, fehlt es den meisten Unternehmen an fachlicher Expertise und vor allem an politischer Legitimität. Diese mangelnde Expertise und Legitimität entbindet Unternehmen aber nicht von ihrer Verantwortung. Um dieser Verantwortung nachkommen zu können, schlage ich drei Maßnahmen vor, welche die pharmazeutischen Unternehmen jetzt vornehmen müssten.

\section{Maßnahmen zum Umgang mit der absehbaren Impfstoffknappheit}

Eine aufkommende Knappheit des noch zu entwickelnden Impfstoffes ist absehbar und scheint mir aufgrund der unzureichenden globalen Produktionskapazitäten auch unvermeidbar zu sein. Die globale Pharmaindustrie produziert u. a. aus Effizienzgründen immer an der Grenze ihrer Kapazitäten. Eine Erweiterung dieser Produktionskapazitäten ist nicht ohne Weiteres und jedenfalls nur langsam möglich - die Errichtung einer entsprechenden Produktionsstätte bedarf im Regelfall mindestens drei Jahre und durchläuft über fünfhundert Sicherheits- und Qualitätskontrollschritte (vgl. Plotkin et al. 2017; Khamsi 2020). Um die zu erwartende Knappheit so gering wie möglich zu halten, müssen sich einzelne Unternehmen auch wenn sie im Normalfall im Wettbewerb miteinander stehen - darauf vorbereiten, ihre bestehenden Produktionskapazitäten zu kombinieren (Pooling). Die Notwendigkeit dieser Maßnahme ergibt sich aus dem o. a. Rechenbeispiel: Selbst einer der global größten Impfstoffhersteller bräuchte bei gezielter Vollauslastung aller Produktionskapazitäten (ceteris paribus) ca. acht Jahre, eine exemplarische globale Nachfrage von acht Milliarden Impfstoffdosen zu befriedigen. ${ }^{6}$

Um ein Pooling der bestehenden Produktionskapazitäten von Unternehmen zu ermöglichen, bedarf es einer Anpassung des in der Europäischen Union und anderswo bestehenden Kartellrechts, das miteinander im Wettbewerb stehenden Firmen weitgehende Kooperationen untersagt. Diese eigentlich zur Vorbeugung von Kartellen und zugunsten von KonsumentInnen entwickelten Regularien sind im vorliegenden Fall kontraproduktiv und müssen entsprechend adaptiert werden. ${ }^{7}$ Pharmaunternehmen sollten ihre Lobbying-Kapazitäten dafür nutzen, politische Entscheidungsträger von der Notwendigkeit der Änderung dieser Wettbewerbsregelungen für diesen konkreten Fall zu überzeugen.

6 Ich weise nochmals darauf hin, dass es sich hier lediglich um eine Beispielrechnung handelt. Die voraussichtliche Knappheit mag geringer oder auch höher ausfallen. Dies ist von einer Reihe von Faktoren, beispielsweise von der Art des zu entwickelnden Impfstoffes, der Notwendigkeit von Mehrfachimpfungen etc., abhängig.

7 Bezüglich einer Reform des Kartellrechts vgl. ebenfalls Crouch (2004; 2013). Die Europäische Kommission adressiert diesen wichtigen Punkt bereits in ihrer Ankündigung vom 17. Juni 2020 (vgl. EC 2020) 
Zweitens sollten die an der Impfstoffentwicklung beteiligten Unternehmen eine Nicht-Patentierung des Impfstoffes in Betracht ziehen oder zumindest einen wenig restriktiven Vollzug der entsprechenden Intellectual Property Rights (IPR). Mit dieser Maßnahme würde explizit eine Nachahmung und Nutzung, also auch eine globale Produktion, ohne juristische Hürden erlaubt und eine schnelle globale Verfügbarkeit von Impfstoffen erreicht werden. Diese drastische Maßnahme könnte außerdem dazu beitragen, die Unternehmen der pharmazeutischen Industrie vor erheblichen Reputations- und Legitimationsschäden sowie vor politischer Einflussnahme zu schützen.

Drittens sollen die Unternehmen der pharmazeutischen Industrie bereits jetzt, während der Entwicklungsphase der Impfstoffe, bestehende globale Institutionen, etwa die WHO, die den Einkauf und die Vergabe der Impfstoffe koordinieren kann, aktiv stärken. Es sind ausschließlich diese Multi-Stakeholder-Plattformen, die die fachliche Expertise sowie zumindest ein Mindestmaß an politischer Legitimität besitzen, um eine nach möglichst ausgeglichenen Interessen sowie an ethischen Kriterien orientierte globale Allokation ebenso wie Gerechtigkeit und Fairness zu gewährleisten.

\section{Zusammenfassung und Diskussion}

Ich argumentiere in diesem Artikel, dass eine marktwirtschaftliche, also letztlich über Preise koordinierte Allokation voraussichtlich äußerst knapper >CoronaImpfstoffe < die globale und soziale Ungleichheit verstetigen und u. U. sogar verschärfen könnte.

Um diese Knappheit zumindest etwas abfedern zu können, sollten die globalen Pharmaunternehmen miteinander kollaborieren, ihre bestehenden Produktionskapazitäten kombinieren und vorbeugend für den reibungslosen - also auch juristisch möglichen - Ablauf solcher Maßnahmen Lobbyarbeit leisten (siehe etwa die neu geschaffene Kooperation zwischen Sanofi und GlaxoSmithKline, vgl. Fröndhoff/Telgheder 2020, und Pfizers Bekenntnis zu Kooperationen und gegebenenfalls zur Verfügbarmachung der Produktionskapazitäten, vgl. Pfizer 2020).

Ich schlage außerdem vor, dass jenes Unternehmen, das als erstes erfolgreich einen Impfstoff entwickelt, in Erwägung ziehen sollte, entsprechende Intellectual Property Rights (IPR) wenig restriktiv zu nutzen oder sogar vollständig aufzugeben und den Impfstoff als Common Good zu betrachten. ${ }^{8}$ Dieser Vorschlag ist zumindest kontrovers und ruft vermutlich unmittelbaren Widerstand bei Rezipienten aus der Pharmaindustrie hervor. Es besteht der (meiner Meinung nach berechtigte) Einwand, dass Unternehmen für ihren Entwicklungsaufwand entsprechend entschädigt werden sollen. Der Entwicklungsaufwand eines Impfstoffes

8 AstraZeneca bietet etwa an, einen möglichen Impfstoff zu Selbstkosten während einer akuten Pandemiephase abzugeben, spezifiziert aber nicht, wie eine solche Phase definiert ist (vgl. Ahmed 2020). 
beträgt durchschnittlich über eine Milliarde Euro (vgl. Waye et al. 2013). Sollte ein Unternehmen nicht wenigstens die anfallenden Entwicklungskosten durch den Verkauf der Impfstoffe zurückerhalten, verliert es voraussichtlich mittelfristig seine kompetitive Basis. Überdies gehen für dieses, aber auch für andere Unternehmen die Anreize verloren, zukünftig Impfstoffe und Medikamente (etwa für den Fall einer Folgeepidemie) zu entwickeln. ${ }^{9}$

Dennoch bestehen für das entsprechende Unternehmen und ebenso für die gesamte pharmazeutische Industrie ebenfalls erhebliche Anreize, diese Option gründlich zu prüfen. Das erfolgreiche Pharmaunternehmen - und mit ihm die gesamte marktwirtschaftlich orientierte Pharmaindustrie - befindet sich in einem Dilemma: Einerseits erhält das Unternehmen, das zuerst erfolgreich einen Impfstoff entwickelt, ein enormes globales Absatzpotenzial mit ebenfalls enormen Preishebeln. Andererseits steht das Unternehmen und mit ihm die gesamte Pharmaindustrie vor einem möglichen und ggf. beträchtlichen Reputations- und Legitimationsproblem. Sollte der Eindruck entstehen, dass das entsprechende Unternehmen nicht alle Maßnahmen ergreift - diese inkludieren die Freigabe der IPR und sollte ein partikulares Pooling der Industrieressourcen nicht ausreichend sein, den Impfstoff schnell und vor allem in den benötigten Mengen zur Verfügung zu stellen, wird es mit erheblichen Reputations- und Legitimationsschwierigkeiten konfrontiert werden. Es wird kaum vermittelbar sein, dass grundsätzlich ein Impfstoff gegen ein Virus existiert, das die größte Gesundheits-, Wirtschafts- und Gesellschaftskrise der letzten hundert Jahre hervorruft, dieser aber aufgrund von unternehmerischen Partikularinteressen nur langsam und zu hohen Preisen verfügbar gemacht wird.

Das aus einem solchen Szenario folgende Reputations- und Legitimationsproblem würde sich nicht nur auf das betroffene Unternehmen beschränken, sondern die gesamte Pharmaindustrie betreffen: Bestehende Vorurteile gegen diese Industrie als ein Konglomerat rücksichtsloser Profitmaximierer würden bestätigt und absehbar zu politischem Zuspruch mit entsprechenden Konsequenzen führen, beispielsweise zu Eingriffen in das Patentrecht, Compulsory Licensing auch über die Corona-Krise hinaus, ggf. sogar Verstaatlichung. Es liegt deshalb auch im Eigeninteresse des einzelnen Unternehmens, aber vielleicht noch stärker im kollektiven Eigeninteresse der Pharmaindustrie, mit ihren zahlreichen starken Assoziationen (zum Beispiel EFPIA, IFPMA, phRMA) ein solches Szenario zu verhindern. Damit eine schnelle globale Versorgung mit Impfstoffen gewährleistet wird, um die eigene Reputation und Legitimation zu gewährleisten und um den genannten politischen Szenarien zuvorzukommen, sollte die Pharmaindustrie neben dem Pooling

9 Der Vorschlag einer Nicht-Patentierung (vgl. auch Scholz/Smith, 2020) widerspricht den Grundprinzipien der ökonomischen Basis der Pharmaindustrie und ist entsprechend kontrovers. Alternativ könnte die Produktion in einer kostenlosen, aber zeitlich begrenzten Lizenz in Betracht gezogen werden. Nachdem die akute Pandemie beendet ist, könnten jene Unternehmen, die hohe unternehmerische Risiken eingegangen sind und teilweise viel eigenes Kapital investiert haben, entsprechend entschädigt werden. 
der verfügbaren Produktionskapazitäten auch die unter anderen Umständen undenkbare Option der partiellen oder vollständigen Aufgabe der IPR eines Corona-Impfstoffes prüfen. Für die Unternehmen der Pharmaindustrie liegen in der aktuellen Krise jedenfalls gleichzeitig enorme Chancen und große Herausforderungen. Wenn die pharmazeutischen Unternehmen zeigen können, dass sie sich während der Krise verantwortlich verhalten, wird dies von politischen Entscheidungsträgern und anderen Stakeholdern voraussichtlich honoriert werden und ihre gesellschaftliche Legitimation steigern. Sollte diese Industrie ihre Entscheidungen allerdings primär an finanziellen Motiven orientieren, wird dies mit spürbaren negativen Reputations- und Legitimationseffekten sowie absehbar mit politischem Druck einhergehen.

Vor dem Hintergrund der oben gezeigten negativen Konsequenzen einer ausschließlichen Koordination über den Preismechanismus und um die oben angeführten Partikularinteressen der Nationalstaaten ausgleichen zu können (vgl. auch Lexchin 2020), müssen andere Wege zur Allokation des Impfstoffes gefunden bzw. ausgebaut werden. Den Pharmaunternehmen kommt in der Allokationsfrage eine wesentliche Mitverantwortung zu, da sie anderweitig nicht von einer fairen und gerechten Verteilung ausgehen können (Wettbewerb zwischen den Nationalstaaten, globales Governance Gap). Gleichzeitig fehlt es den Unternehmen an Expertise (beispielsweise bezüglich sozialer und globaler Ungleichheitsphänomene) und an Legitimität, allein entscheiden zu können, wer wann und zu welchem Preis Impfstoffe erhält. Deshalb bezieht sich mein dritter Vorschlag auf die Stärkung solcher Institutionen, die Multi-Stakeholder-Diskurse hinsichtlich der Koordination der Allokation eines Impfstoffes beherbergen können. ${ }^{10}$ In solchen Multi-Stakeholder-Dialogen kann zumindest versucht werden, die Interessen möglichst vieler Betroffener abzubilden, bestehende Machtasymmetrien (bargaining power) möglichst zu nivellieren und die Allokation an anderen konkreten Kriterien als dem Preis festzumachen (etwa Bedürftigkeit: Ausmaß der Betroffenheit von Territorien, Betroffenheit einzelner Kommunen, Ausmaß des Risikos für bestimmte Bevölkerungsgruppen, v. a. nach bestehenden Vorerkrankungen, Alter etc., aber auch in Hinblick auf bestehende Medikation bei bestimmten Gruppen, etwa Menschen mit Autoimmunerkrankungen, die unter Immunsupressiva-Therapie stehen; Gerechtigkeit: in Hinblick auf politische und kulturelle Teilhabe, ökonomische Entwicklungschancen, auch vor dem Hintergrund bestehender sozialer Ungleichheit).

Die sofortige Stärkung einer Institution (zum Beispiel der WHO), die als Plattform für diesen Dialog geeignet ist, liegt nicht nur in der gesellschaftlichen und quasipolitischen Verantwortung der pharmazeutischen Unternehmen, sondern ebenfalls in ihrem Eigeninteresse. Die global agierenden Pharmaunternehmen soll-

10 Eine von der WHO koordinierte Allokation von Medikamenten und Impfstoffen ist nicht unüblich, vgl. beispielsweise das Pandemic Influenza Preparedness Framework (PIP) (WHO 2020b). 
ten davon ausgehen, dass ihre globale Reputation und Legitimation davon abhängen, wie der globale Allokationsprozess eines Corona-Impfstoffes gestaltet wird. $\mathrm{Da}$ sie sich bezüglich einer verantwortungsbewussten Allokation - welche zum Schutz der eigenen Legitimation und Reputation unerlässlich ist - weder auf ihre eigene Expertise noch auf den Preismechanismus oder auf die von Partikularinteressen getriebenen Nationalstaaten verlassen können, liegt ihre einzige Hoffnung in einer starken supranationalen Institution. Eine verantwortungsbewusste Preisgestaltung, Produktion und Allokation eines >Corona-Impfstoffes< ist nicht nur aus Gründen der gesellschaftlichen Verantwortung Aufgabe der pharmazeutischen Unternehmen; die Moderationen dieser Prozesse an eine supranationale Institution teilweise zu delegieren, liegt ebenfalls in ihrem Eigeninteresse. ${ }^{11}$

\section{Literaturverzeichnis}

Abmed, A. K. (2020): Oxford, AstraZeneca Covid-19 Deal Reinforces ,Vaccine Sovereignty`. We Need a People's Vaccine Instead. Link: https://www.statnews.com/2020/06/04/oxfordastrazeneca-covid-19-deal-reinforces-vaccine-sovereignty/ (last access on July 24th, 2020).

Becker, M./Müller, P. (2020): Keiner für alle, alles für einen: Die EU will geschlossen gegen die Corona-Epidemie kämpfen - doch beim Treffen der Gesundheitsminister zeigte sich: Kommt das Virus, ist sich jeder selbst der Nächste. Link: https://www.spiegel.de/politik/de utschland/coronavirus-wie-die-solidaritaet-der-eu-staaten-in-der-krise-broeckelt-a-6c8ae65 9-d717-4f8d-91e5-223c4505ab53 (zuletzt abgerufen am 26.05.2020).

Bubrowski, H. (2020): Forscherdrang und Machtkampf. Link: https://www.faz.net/aktuell/poli tik/inland/wird-die-welt-einen-corona-impfstoff-solidarisch-teilen-16777577.html (zuletzt abgerufen am 26.05.2020).

Crouch, C. (2004): Post-Democracy, Cambridge, Malden, MA: Polity Press.

Crouch, C. (2013): The Strange Non-Death of Neoliberalism, Cambridge: Polity.

Dostert, E. (2020): Curevac-Eigner Hopp äußert sich erstmals zur Trump-Offerte. Link: https:/ /www.sueddeutsche.de/wirtschaft/curevac-hopp-coronavirus-1.4847048 (zuletzt abgerufen am 26.05.2020).

EC (2020): Coronavirus: Commission Unveils EU Vaccines Strategy. Link: https://ec.europa.eu/ commission/presscorner/detail/en/ip_20_1103 (last access on July 24th, 2020).

Fisher, M./Bubola, E. (2020): As Coronavirus Deepens Inequality, Inequality Worsens Its Spread. Link: https://www.nytimes.com/2020/03/15/world/europe/coronavirus-inequality. $\mathrm{html}$ (last access on May 26th, 2020).

Friedman, M. (1962): Capitalism and Freedom, Chicago, IL: University of Chicago Press.

Friedman, M. (1970): The Social Responsibility of Business is to Increase Its Profits, in: The New York Times Magazine, September 13, 1970.

Fröndhoff, B./Telgheder, M. (2020): Pharmariesen bündeln ihre Kräfte gegen Covid-19. Link: https://www.handelsblatt.com/unternehmen/industrie/neue-allianz-pharmariesen-buendeln -ihre-kraefte-gegen-covid-19/25684902.html (zuletzt abgerufen am 26.05.2020).

Garde, D./Silverman, E. (2020): Less Than a Movie Ticket or >Impossible to Overpay<? Experts Name Their Price for Remdesivir. Link: https://www.statnews.com/2020/05/15/gil ead-remdesivir-pricing-coronavirus/ (last access on July 24th, 2020).

Khamsi, R. (2020): If a Coronavirus Vaccine Arrives, Can the World Make Enough?, in: Nature, Vol. 580/No. 7805, 578-580.

11 Ich danke Maria Riegler für die redaktionelle Unterstützung bei diesem Beitrag. 
Klingst, M. (2020): Beschleuniger der Ungleichheit. Link: https://www.zeit.de/politik/ausland/2 020-05/covid-19-ungleichheit-diskriminierung-rassismus-minderheiten-corona-5vor 8 (zuletzt abgerufen am 26.05.2020).

Landfried, C. (2020): Das Virus kennt keine Schlagbäume. Link: https://www.faz.net/aktuell/fe uilleton/das-virus-kennt-keine-schlagbaeume-was-die-eu-nun-unternehmen-muss-1670880 7.html (zuletzt abgerufen am 26.05.2020).

Le Ker, H./Müller, M. U. (2020): Was die Impfstoffherstellung so schwierig macht. Link: https: //www.spiegel.de/wirtschaft/unternehmen/coronavirus-was-die-impfstoffherstellung-so-sch wierig-macht-a-f1365987-0a22-4358-be3c-b3d15e9bc0ad (zuletzt abgerufen am 26.05.2020).

Lexchin, J. (2020): As U.S. Buys Up Remdesivir, >Vaccine Nationalism< Threatens Access to Covid-19 Treatments. Link: https://theconversation.com/as-u-s-buys-up-remdesivir-vaccine -nationalism-threatens-access-to-covid-19-treatments-141952 (last access on July 24th, 2020).

Loerzer, S. (2020): Eine Bugwelle an Fällen: In den Sozialbürgerhäusern ist man bislang kreativ mit Corona umgegangen. Mit den Lockerungen wird nun die Not sichtbar, die durch die Krise entstanden ist. Link: https://www.sueddeutsche.de/muenchen/corona-muenchen-sozi albuergerhaeuser-faelle-1.4917196 (zuletzt abgerufen am 26.05.2020).

Mintzes, B./Hoen, E. (2020): The US Has Bought Most of the World's Remdesivir. Here's What It Means for the Rest of Us. Link: https://theconversation.com/the-us-has-bought-m ost-of-the-worlds-remdesivir-heres-what-it-means-for-the-rest-of-us-141791 (last access on July 24th, 2020).

Mullard, A. (2020): COVID-19 Vaccine Development Pipeline Gears Up, in: The Lancet, Vol. 395/No. 10239, 1751-1752.

Neuhäuser, C. (2011): Unternehmen als moralische Akteure, Berlin: Suhrkamp.

Orts, E. W./Smith, N. C. (Hrsg.) (2017): The Moral Responsibility of Firms, Oxford: Oxford University Press.

Pfizer (2020): Pfizer Outlines Five-point Plan to Battle Covid-19. Link: https://www.pfizer.com /news/press-release/press-release-detail/pfizer_outlines_five_point_plan_to_battle_covid_19 (last access on July 24th, 2020).

Plotkin, S./Robinson, J. M./Cunningham, G./Iqbal, R./Larsen, S. (2017): The Complexity and Cost of Vaccine Manufacturing - an Overview, in: Vaccine, Vol. 35/No. 33, 4064-4071.

Scholz, M./Smith, N. C. (2020): In the Face of a Pandemic: Can Pharma Shift Gears? Link: https://sloanreview.mit.edu/article/in-the-face-of-a-pandemic-can-pharma-shift-gears/ (last access on July 24th, 2020).

UNAIDS (2020): Uniting Behind a People's Vaccine Against COVID-19. Link: https://www.un aids.org/en/resources/presscentre/featurestories/2020/may/20200514_covid19-vaccine-ope n-letter (last access on July 24th, 2020).

Usher, A. D. (2020): COVID-19 Vaccines for All?, in: The Lancet, Vol. 395/No. 10240, 18221823.

Waye, A./Jacobs, P./Schryvers, A. B. (2013): Vaccine Development Costs: A review, in: Expert Review of Vaccines, Vol. 12/No. 12, 1495-1501.

WHO (2020a): Draft Landscape of COVID-19 Candidate Vaccines. Link: https:/www.who.in t/who-documents-detail/draft-landscape-of-covid-19-candidate-vaccines (last access on July 24th, 2020).

WHO (2020b): Pandemic Influenza Preparedness (PIP) Framework. Link: https://www.who.int /influenza/pip/en/ (last access on July 24th, 2020).

Wimalasena, J. (2020): »Dann werde ich wohl auf dem Rücksitz meines Autos schlafen müssen «: Millionen Amerikaner können in der Corona-Krise ihre Miete nicht zahlen. Es drohen massenhaft Räumungen. Etwa im armen Oklahoma, wo die Gerichte jetzt wieder öffnen. Link: https://www.zeit.de/wirtschaft/2020-05/zwangsraeumungen-usa-corona-wirtsch aftskrise-obdachlosigkeit (zuletzt abgerufen am 26.05.2020). 
